\title{
Integrated Control and Switching Strategy for a Grid-Connected Modular Multilevel Converter
}

\author{
Yan Ma, Student Member, IEEE, Lingling Fan, Senior Member, IEEE, Zhixin Miao, Senior Member, IEEE
}

\begin{abstract}
This paper presents an integrated control and switching scheme for a grid-connected modular multilevel converter (MMC). The control consists of the conventional vector control (outer PQ control, inner line current control) used in two-level voltage source converters (VSCs) and additional circulating current mitigation control. Circulating currents in a modular multilevel converter (MMC) will be eliminated using a proportional resonant controller. The generated upper and lower arm reference voltages will be sent to the switching sequence generating blocks to generate gate signals to submodules. Phaseshifted PWM scheme will be used to determine how many submodules to be turned on in an arm while the voltage balancing block will determine which submodules to be turned on. Matlab/SimPowerSystems simulation environment is used to model a grid-connected MMC with the integrated PQ control. Simulation case studies demonstrate the effectiveness of the proposed control strategy. The contribution of this paper lies in the modeling and presentation of the entire control and switching procedure for a grid-connected MMC, with reference to the control of two-level VSCs. This paper will provide readers with a clear understanding of the difference between MMC control and two-level VSC control.
\end{abstract}

Index Terms-Modular Multilevel Converter, Circulating Current Suppression Control, MATLAB/Simpowersystem, Proportion Resonance Controller

\section{INTRODUCTION}

$\mathbf{H}$ IGH voltage direct current (HVDC has been applied widely to increase the capability for power transmission and link different ac networks together [1]. Normally, an HVDC system can be either voltage-source converter (VSC) based or current-source converter based [2]. VSC-based HVDC systems can control the active and reactive power separately. Unlike the conventional CSC-based HVDC systems where acres of land are used for filter installation, VSC-HDVC requires only small sized filters on the ac side of the converter.

Compared with a two-level VSC, multilevel voltage-source converters have much less harmonics in the output voltage, which significantly reduces the size of grid-side filters [4]. Among the different multilevel converters, modular multilevel converter (MMC) has extensibility for several hundreds of output voltage levels. Therefore, MMC is ideal for highvoltage high-power applications, e.g., HVDC transmission [5], high-voltage motor drives [6], and electric railways [7]. Fig. 1 is the topology of a three-phase MMC. For an $N+1$ level MMC, there are $N$ submodules on each arm of the converter. Each submodule is a half bridge dc-dc converter.

L. Fan, Z.Miao and Y. Ma are with Dept. of Electrical Engineering, University of South Florida, Tampa FL 33620. Emails: linglingfan@usf.edu, zmiao@usf.edu and yanma@mail.usf.edu Phone: (813)974-2031.

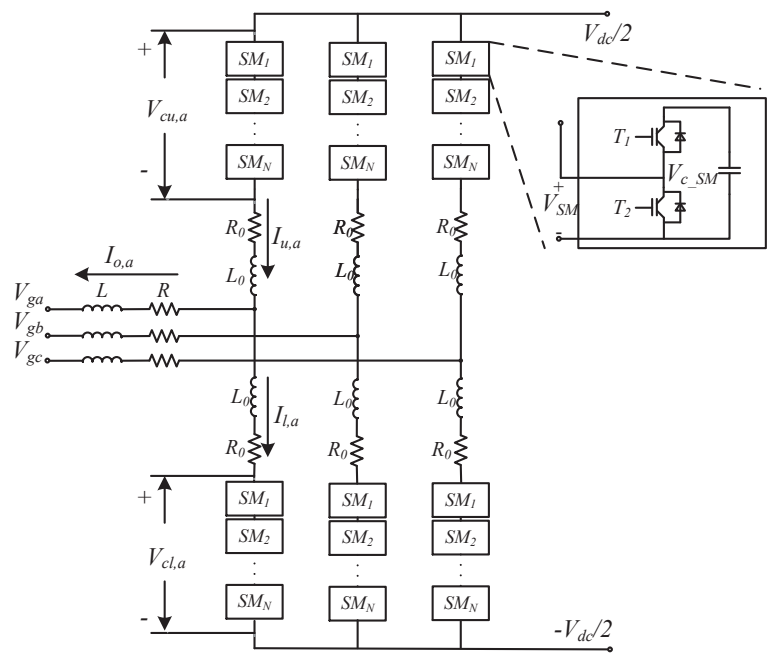

Fig. 1. Three phase MMC topology.

MMC control differs from two-level VSC control in two aspects: (i) switching sequence generation; and (ii) the inclusion of circulating current mitigation control.

In switching sequence generation, in two-level VSCs, the output from PWM is the switching sequence directly fed to the gates. In MMCs, due to the large number of submodules, the output of PWM or other types of switching schemes, is the number of submodules to be turned on at each arm. Which submodules to be turned on depends on additional submodule voltage balance consideration. The PWM switching schemes are also very different from those of two-level VSCs. Phasedisposition (PD)-PWM and phase shifted (PS)-PWM are often adopted in MMC [8]. In MMC's PWM, there are usually many carrier signals for the reference sinusoidal signal to be compared to; while in two-level VSC's PMW, there is usually one triangular carrier signal.

Due to difference in the arm voltages in the three bridges, there are circulating currents flowing within the MMC [9]. Although the circulating current has no effect on output voltages and currents, it increases the RMS value of the arm current, which raises the required size of the power electronic devices and increases the converter losses [10]. Circulating current can be reduced by increasing the size of the arm inductors or adding extra capacitors across each phase. However, the hardware approach can not completely eliminate circulating current. In addition, it is not economical due to the high cost of high-power inductors. Therefore, it is necessary to investigate and design a control strategy to suppress the 
circulating current.

It has been proved that circulating current have a dc component and a double frequency component at steady state [11], [12]. A control strategy that converts the ac component of circulating current into de signal via $a c b$ to $d q$ transform, has been developed in [9]. However, when the circulating current contains additional harmonics with different frequency, additional $a b c / d q$ transformation is required, which makes the controller very complex. Instead, control schemes without $a b c / d q$ or $a c b / d q$ have been adopted in the literature. [13] has presented a circulating current suppression strategy with a PR controller for a passive load connected MMC. [14] and [15] control the MMC via model predictive control (MPC), while MPC requires high amount calculation volume of the processor.

The objective of this paper is to present an integrated MMC control for a grid-connected converter. The control consists of the conventional vector control (outer PQ control, inner line current control), circulating current mitigation control, phase-shifted PWM scheme and submodule voltage balancing block. Proportional resonant (PR) controller will be used to eliminate the ac components in the circulating currents. The PR controller controls the circulating current in each phase separately, so it can be applied to a single phase or any other number of phases MMC directly.

This paper is structured as follows. Section II explains the basic operation of MMC and the gate signal generating blocks. Section III explains the control strategy for circulating current suppression control (CCSC), and vector control including real/ reactive power control and current control. A case study and simulation results are presented in Section IV followed by conclusions in Section V.

\section{BASIC OPERATION OF MMC}

\section{A. Basic Structure}

Fig. 1 shows the overall structure of a three-phase MMC consisting of six arms. Subscripts $u$ and $l$ denote upper and lower arms, respectively. There are $N$ sub-modules and one inductor $L_{0}$ on each arm. A resistor $R_{0}$ is inserted to represent the switching loss of the IGBTs on each arm. The output voltage of each sub-module has two values, $U_{c}$ (when $T_{1}$ is connected) and 0 (when $T_{2}$ is connected). When the number of sub-modules or the switching frequency is high enough, the voltage across whole sub-modules in each arm can be considered as continuous. Since the dc side capacitors are usually big enough, the voltage across the arm can be considered as constant dc voltage sources. Thus, we can express a single phase-equivalent circuit of a MMC as Fig.2.

\section{B. Plant models for ac line current control and circulating current control}

In Fig. 2, $i_{u}$ and $i_{l}$ are the arm currents for upper and lower arms; $i_{o}$ and $v_{o}$ are the converter output current and voltage respectively. The circulating current flowing within the converter is denoted as $i_{\text {diff }}$. Since the upper and lower arm are symmetric, ideally both lower and upper arm currents contain

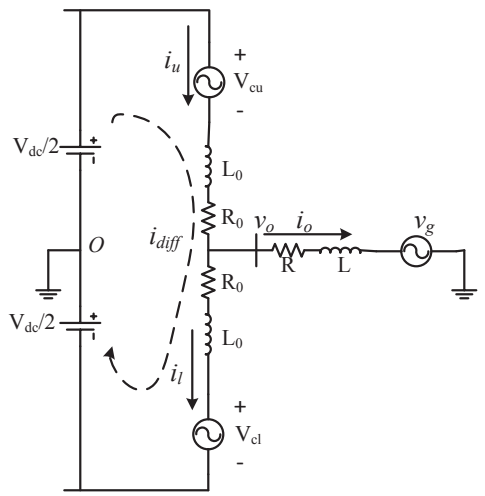

Fig. 2. Single phase equivalent circuit of MMC.

half of the converter output current. Therefore, with KCL, we can get following equations:

$$
\left\{\begin{array} { l } 
{ i _ { u } = i _ { \mathrm { diff } } + \frac { i _ { o } } { 2 } } \\
{ i _ { l } = i _ { \mathrm { diff } } - \frac { i _ { o } } { 2 } }
\end{array} \Rightarrow \left\{\begin{array}{l}
i_{\mathrm{diff}}=\frac{i_{u}+i_{l}}{2} \\
i_{o}=i_{u}-i_{l} .
\end{array}\right.\right.
$$

The voltage across the arm resistance and inductance can be expressed by the arm current. Therefore, with KVL, we can have the voltage relationship as follow:

$$
\left\{\begin{array}{l}
v_{u}+i_{u} R_{0}+L_{0} \frac{\mathrm{d} i_{u}}{\mathrm{~d} t}=\frac{V_{\mathrm{dc}}}{2}-v_{o} \\
v_{l}+i_{l} R_{0}+L_{0} \frac{\mathrm{d} i_{l}}{\mathrm{~d} t}=\frac{V_{\mathrm{dc}}}{2}+v_{o}
\end{array}\right.
$$

Considering that the output voltage $v_{o}$ can be written as $v_{g}+i_{o} R+L \frac{\mathrm{d} i_{o}}{\mathrm{~d} t}$ and (1), by substracting the two equations from (2) we have:

$$
\frac{v_{u}-v_{l}}{2}+\left(R+\frac{1}{2} R_{0}\right) i_{o}+\left(L+\frac{1}{2} L_{0}\right) \frac{\mathrm{d} i_{o}}{\mathrm{~d} t}+v_{g}=0
$$

It is obvious that the term $\frac{v_{u}-v_{l}}{2}$ in (3) drives the output current of the converter, therefore we name this term as $e$, which is the inner emf of the converter. We can have an equivalent circuit of MMC as Fig. 3, which is the plant model of inner current control loop of an MMC.

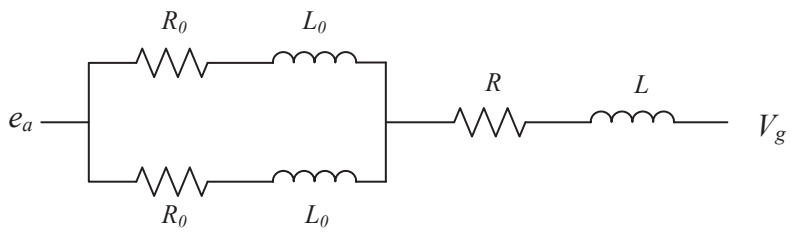

Fig. 3. An equivalent circuit of one phase of MMC.

Adding the two equations in (2) leads to the plant model of the circulating current control:

$$
i_{\text {diff }} R_{0}+L_{0} \frac{\mathrm{d} i_{\mathrm{diff}}}{\mathrm{d} t}=\frac{V_{\mathrm{dc}}}{2}-\frac{v_{u}+v_{l}}{2} .
$$

The term $\frac{V_{\mathrm{dc}}}{2}-\frac{v_{u}+v_{l}}{2}$ in (4) is the voltage drives the circulating current. We name this voltage as $e_{\text {diff }}$. Considering the definitions of $e$ and $e_{\mathrm{diff}}$, we can have

$$
\left\{\begin{array}{l}
v_{u}=\frac{V_{\mathrm{dc}}}{2}+e-e_{\mathrm{diff}} \\
v_{l}=\frac{V_{\mathrm{dc}}}{2}-e-e_{\mathrm{diff}}
\end{array}\right.
$$




\section{Switching Scheme}

Through the vector control, $e_{a b c}$ will be found. Through the circulating current suppression control $e_{\mathrm{diff}}$ for three phases will be found. Applying (5), then the upper and lower arm voltage levels $\left(v_{u}\right.$ and $\left.v_{l}\right)$ can be found. The driving scheme generates gate signals for each submodule in MMC given $v_{u}$ and $v_{l}$. A PS-PWM [9] as shown in Fig. 4 will generate the number of sub-modules needed to be switched on. Unlike a two-level VSC, $N$ triangular signals will be used, each shifting from the previous one by $360^{\circ} / N$. The sinusoidal reference signal will be compared with the carrier signals. The output from the PWM is the number of submodules to be switched on $\left(N_{\text {on }}\right)$.

From (5), when the term $e_{\text {diff }}$ is not zero, the summation of $v_{u}$ and $v_{l}$ does not equal to $V_{d c}$. Therefore, the reference voltage signals of the lower and upper arms of each phase should be given separately. There are six reference signals for a three-phase MMC. After PWM, a capacitor voltage balancing block selects the proper sub-modules to be switched. The algorithm of the capacitor voltage balancing block is shown in Fig. 5. This algorithm is basically to a sorting algorithm to determine which submodules to be switched on. For upper arms, when the arm current is positive or charging, then the submodules with the lowest voltages should be turned on. The sorting algorithm can balance the submodule capacitor voltage and has been applied in our previous paper on MMC switching scheme design [15].

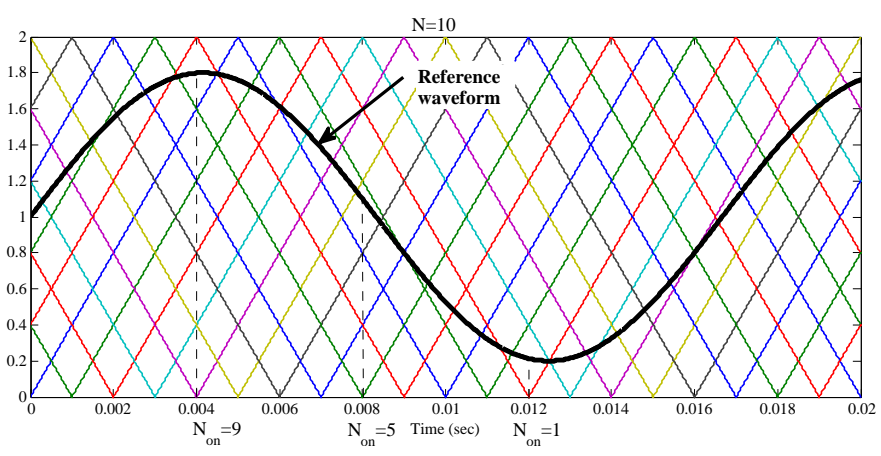

Fig. 4. Modified PS-PWM scheme to determine how many submodules will be switched on in an arm.

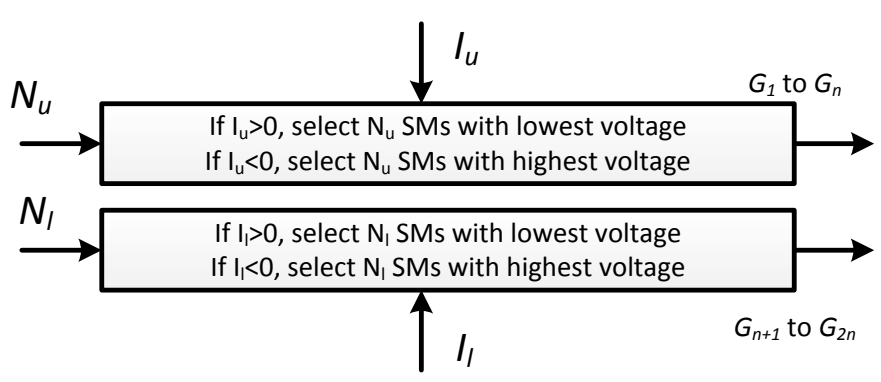

Fig. 5. Capacitor voltage balancing block.

\section{MMC CONTROLS: CirCUlating CURRENT SUPPRESSION, INNER CURRENT CONTROL, AND PQ CONTROL}

The circulating current has no effect on the output of MMC. From (4) and (3), it is obvious that converter output current control and circulating current control can be separated into independent control loops. [11] has proved that the ac component of the energy stored in each phase of a MMC has a double frequency ac component, which can be written as:

$$
W_{A C, \text { phase }}=\frac{V_{d c} I_{d c}}{6 \omega_{0} \cos \varphi} \sin \left(2 \omega_{0} t+\varphi\right)
$$

where $\varphi$ is the angle difference between converter output voltage and current. The energy is stored in the submodule capacitors. Therefore, there is a double frequency component on the total inserted sub-module output voltage $v_{u}$ and $v_{l}$. And the double frequency of $v_{u}$ and $v_{l}$ causes the circulating current, $i_{\text {diff }}$ to have a double frequency component. Besides the ac component, the dc side current flows through each phase of MMC. Since each phase has the same impedance, the circulating current on each phase contains a dc component of $i_{d c} / 3$.

\section{A. PR Controller}

PR control can achieve high bandwidth at certain resonant frequency. Through PR control, measurement signal can track the reference signal without steady-state error at the resonance frequency. The ideal transfer function of a PR controller is shown in (7).

$$
G_{P R}(s)=K_{P}+\frac{K_{r} s}{s^{2}+\omega_{0}^{2}}
$$

The quasi-PR controller has a better noise rejection and has been implemented in [13] for circulating current mitigation. The transfer function of a quasi-PR controller is shown in (8).

$$
G_{Q P R}(s)=K_{P}+\frac{2 \omega_{c} K_{r} s}{s^{2}+2 \omega_{c} s+\omega_{0}^{2}}
$$

At steady state, circulating current contains only double frequency component, while during the transient there is a quadruple harmonic component in the circulating current. Therefore two PR controllers are connected in parallel to handle both double and quadruple frequency respectively, as shown in Fig. 6.

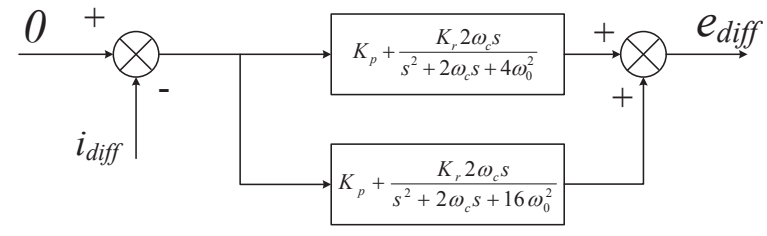

Fig. 6. Two PR controllers in parallel to control double and quadruple component of circulating current.

Fig. 7 is the overall control loop for vector control (outer active/reactive power control, inner current control) combined with circulating current suppression control. The vector control has been used in grid-connected two-level VSC. In MMC 


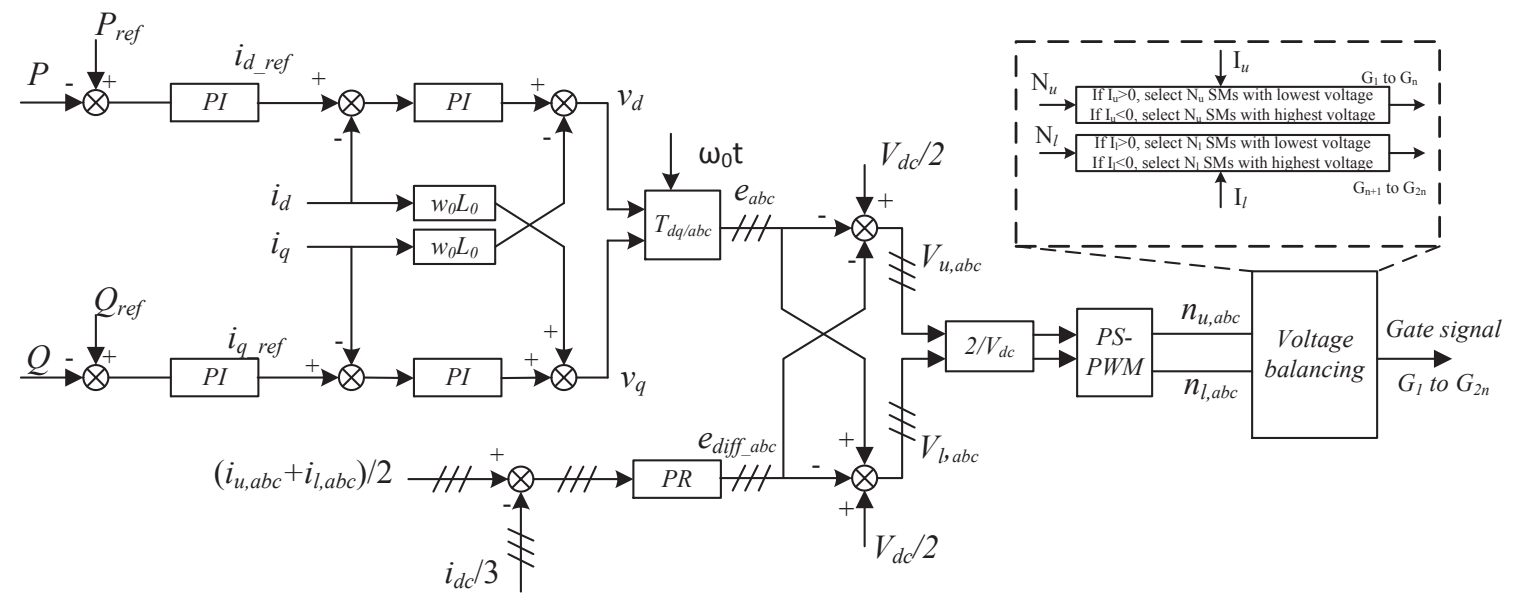

Fig. 7. Integrated MMC Control and Switching.

control, the vector control along with $d q / a b c$ transformation will generate $e_{a b c}$. The circulating current control generates $e_{\text {diff }}$. The upper arm SM voltage $V_{u}$ and $V_{l}$ are determined by (5). The PS-PWM and capacitor voltage balancing blocks are used to generate the gate control signal for every sub-module.

\section{CAse Study And Simulation Results}

A detailed circuit-based simulation model of a grid-connected MMC has been built in MATLAB/SimPowerSystem. Table I shows the parameters of the circuit. Table II gives the controller parameters. The MMC works in real and reactive power control mode. At $t=0.1 \mathrm{~s}, \mathrm{CCSC}$ is activated; then at $t=0.4 \mathrm{~s}$, the reference of real power is reduced to $0.8 \mathrm{pu}$ from $1 \mathrm{pu}$; at $t=0.6 \mathrm{~s}$, the reactive power reference is increased to $0.2 \mathrm{pu}$ from $0.1 \mathrm{pu}$.

TABLE I

Circuit PARAMETERS AND OPERATING CONDITIONS OF THE SIMULATION

\begin{tabular}{l|l|c}
\hline Items & Values & Comments \\
\hline Active power $P$ & $10 \mathrm{MW}$ & $1.0 p . u$. \\
\hline Reactive power $Q$ & $1 \mathrm{MW}$ & $0.1 p . u$. \\
\hline Grid voltage & $20 \mathrm{kV}$ & $V_{\text {peak }} L-N$ \\
\hline Base power $S_{\text {base }}$ & $10 \mathrm{MW}$ & \\
\hline Base voltage $V_{\text {base }}$ & $20 \mathrm{kV}$ & \\
\hline Line inductance $L$ & $50 \mathrm{mH}$ & $0.4712 \mathrm{pu}$ \\
\hline Line resistance $R$ & $0.03 \Omega$ & $7.5 \times 10^{-4} p . u$. \\
\hline Arm inductance $L_{0}$ & $2 \mathrm{mH}$ & $0.0188 p . u$. \\
\hline Arm resistance $R_{0}$ & $0.1 \Omega$ & $0.0025 p . u$. \\
\hline DC bus voltage $V_{d c} / 2$ & $30 \mathrm{kV}$ & $V_{\mathrm{dc}}= \pm 20 \mathrm{kV}$ \\
\hline Number of SMs per arm $N$ & 10 & \\
\hline SM capacitance & $2500 \mu \mathrm{F}$ & \\
\hline SM capacitor voltage & $6000 \mathrm{~V}$ & \\
\hline Sampling time & $25 \mu \mathrm{s}$ & \\
\hline Carrier frequency of PWM & $1000 \mathrm{~Hz}$ & \\
\hline
\end{tabular}

Fig. 8 presents the simulation result of the $\mathrm{AC}$ components of the circulating current. Circulating current control has successfully eliminated the ac components after it was activated at $t=0.2 s$. The ac component of circulating current is limited within $0.1 \mathrm{pu}$ after the activation while it can reach 2 pu before the activation.

The performance of the vector control is demonstrated in Fig. 9. It can be seen that real power and reactive power
TABLE II

CONTROLler Parameters of THE Simulation

\begin{tabular}{|l|l|c|}
\hline Items & Values & comment \\
\hline MMC output power measurement unit & $\mathrm{pu}$ & $S_{\text {base }}=10 \mathrm{MW}$ \\
\hline MMC output current measurement unit & $\mathrm{pu}$ & $I_{\text {base }}=500 \mathrm{~A}$ \\
\hline Circulating current measurement unit & $\mathrm{A}$ & \\
\hline$K_{P}$ for inner current control loop & 10 & \\
\hline$K_{i}$ for inner current control loop & 5000 & \\
\hline$K_{P}$ for outer power control loop & 1 & \\
\hline$K_{i}$ for outer power control loop & 1000 & \\
\hline$K_{P}$ for CCSC & 5 & \\
\hline$K_{r}$ for CCSC & 1000 & \\
\hline$\omega_{c}$ for quasi-RP controller & 0.0005 & \\
\hline
\end{tabular}

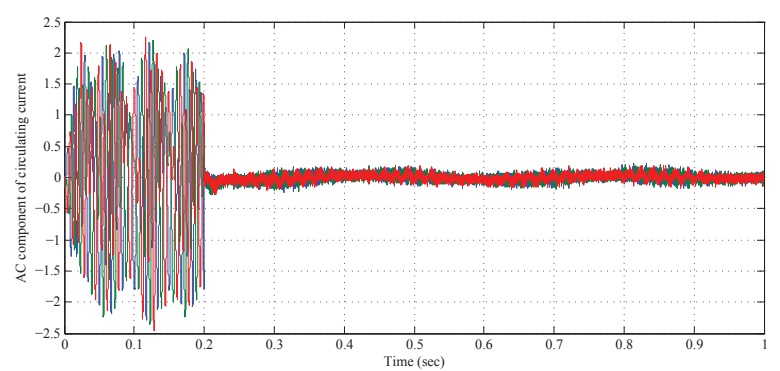

Fig. 8. Circulating current with suppression control activated at $t=0.2 \mathrm{~s}$.

measurements can quickly track the power reference order. In addition, real and reactive power can be controlled respectively.

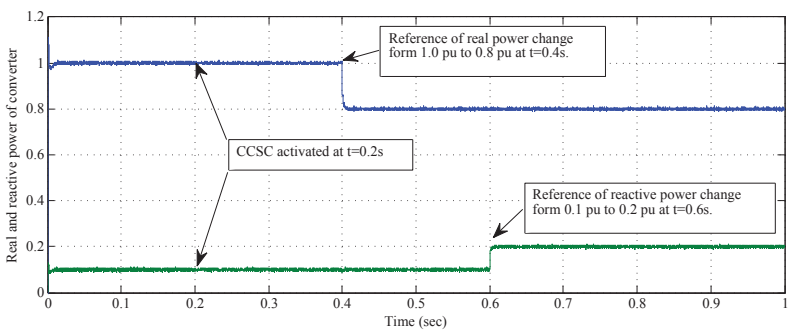

Fig. 9. Output real and reactive power with circulating current suppression control activated at $t=0.2 \mathrm{~s}$.

Fig. 10 shows upper arm current, lower arm current and 
output current of phase a. After $t=0.2 s$, the double frequency component of arm currents are eliminated. Only dc and fundamental frequency components are left on the arm current with some small high order ripples. The output current of converter is not impacted by applying the suppression control.
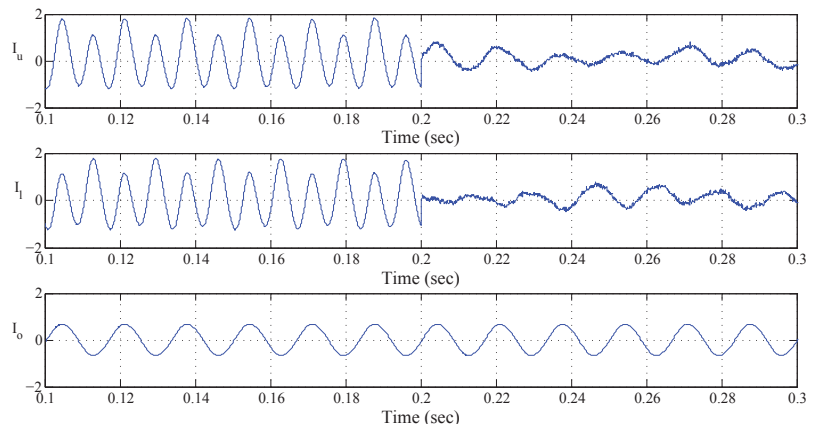

Fig. 10. Upper arm current, lower arm current, and output current with CCSC activated at $t=0.2 \mathrm{~s}$
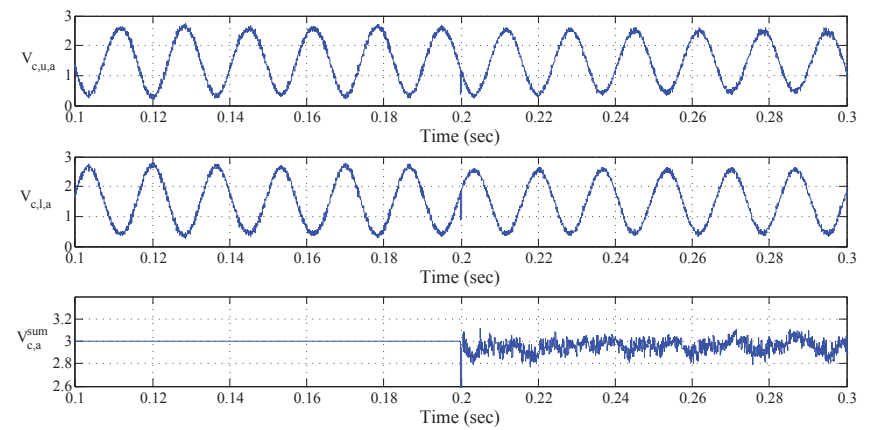

Fig. 11. Upper arm SM voltage, lower arm SM voltage, and whole phase $\mathrm{SM}$ voltage with CCSC activated at $t=0.2 \mathrm{~s}$.

Fig. 11 shows the whole sub-modules (SM) voltage of the upper arm, lower arm, and whole phase. It shows that before CCSC is applied, the summation of whole phase sub-module output voltage is constant at $V_{d c}$. This is because $v_{u}+v_{l}=$ $V_{\mathrm{dc}}+2 e_{\mathrm{diff}}$ (according to (5)), and when there is no CCSC, $e_{\text {diff }}=0$. There is an inductor between the DC voltage source and MMC. After CCSC is applied at $t=0.2 s$, the whole phase sub-module output voltage is no longer a constant value. And that leads to a ripple on dc current. Since CCSC eliminates the ac component of the arm current, the RMS value of arm current is reduced. Therefore, the voltage ripple of sub-module capacitor is also reduced, which is shown in Fig. 12.

\section{CONClusion}

In this paper, operation and plant models of MMC for controls are first analyzed. A control strategy that combines vector control and circulating current suppression control together has been presented. The vector control for MMC is exactly the same as that of a two-level VSC. The circulating current control is achieved by PR controls which handle both double- and quadruple- frequency components of the circulating currents. Switching scheme is also presented. A detailed simulation model has been developed in MATLAB/SimPowerSystem.

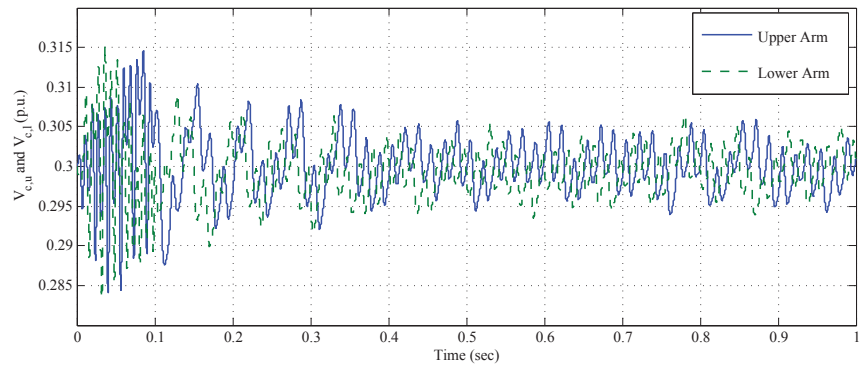

Fig. 12. Upper arm SM capacitor voltage, lower arm SM capacitor voltage with CCSC activated at $t=0.2 s$.

Simulation results demonstrate the effectiveness of the integrated MMC control in active/reactive power tracking and circulating current suppression.

\section{REFERENCES}

[1] Q. Tu, Z. Xu, Y. Chang, and L. Guan, "Suppressing dc voltage ripples of mmc-hvdc under unbalanced grid conditions," Power Delivery, IEEE Transactions on, vol. 27, no. 3, pp. 1332-1338, July 2012.

[2] J.-W. Moon, C.-S. Kim, J.-W. Park, D.-W. Kang, and J.-M. Kim, "Circulating current control in mmc under the unbalanced voltage," Power Delivery, IEEE Transactions on, vol. 28, no. 3, pp. 1952-1959, July 2013.

[3] L. Xu, L. Fan, and Z. Miao, "Modeling and simulation of multi-terminal hvdc for wind power delivery," in Power Electronics and Machines in Wind Applications (PEMWA), 2012 IEEE, July 2012, pp. 1-6.

[4] L. Franquelo, J. Rodriguez, J. Leon, S. Kouro, R. Portillo, and M. Prats, "The age of multilevel converters arrives," Industrial Electronics Magazine, IEEE, vol. 2, no. 2, pp. 28-39, June 2008.

[5] M. Saeedifard and R. Iravani, "Dynamic performance of a modular multilevel back-to-back hvdc system," Power Delivery, IEEE Transactions on, vol. 25, no. 4, pp. 2903-2912, Oct 2010.

[6] M. Hiller, D. Krug, R. Sommer, and S. Rohner, "A new highly modular medium voltage converter topology for industrial drive applications," in Power Electronics and Applications, 2009. EPE '09. 13th European Conference on, Sept 2009, pp. 1-10.

[7] M. Winkelnkemper, A. Korn, and P. Steimer, "A modular direct converter for transformerless rail interties," in Industrial Electronics (ISIE), 2010 IEEE International Symposium on, July 2010, pp. 562-567.

[8] B. Li, R. Yang, D. Xu, G. Wang, W. Wang, and D. Xu, "Analysis of the phase-shifted carrier modulation for modular multilevel converters," Power Electronics, IEEE Transactions on, vol. 30, no. 1, pp. 297-310, Jan 2015.

[9] Q. Tu, Z. Xu, and L. Xu, "Reduced switching-frequency modulation and circulating current suppression for modular multilevel converters," Power Delivery, IEEE Transactions on, vol. 26, no. 3, pp. 2009-2017, July 2011.

[10] A. Antonopoulos, L. Angquist, and H.-P. Nee, "On dynamics and voltage control of the modular multilevel converter," in Power Electronics and Applications, 2009. EPE '09. 13th European Conference on, Sept 2009, pp. $1-10$.

[11] Q. Tu, Z. Xu, H. Huang, and J. Zhang, "Parameter design principle of the arm inductor in modular multilevel converter based hvdc," in Power System Technology (POWERCON), 2010 International Conference on, Oct 2010, pp. 1-6.

[12] L. Harnefors, A. Antonopoulos, S. Norrga, L. Angquist, and H.-P. Nee, "Dynamic analysis of modular multilevel converters," Industrial Electronics, IEEE Transactions on, vol. 60, no. 7, pp. 2526-2537, July 2013.

[13] X. She, A. Huang, X. Ni, and R. Burgos, "Ac circulating currents suppression in modular multilevel converter," in IECON 2012 - 38th Annual Conference on IEEE Industrial Electronics Society, Oct 2012, pp. 191-196.

[14] J. Qin and M. Saeedifard, "Predictive control of a modular multilevel converter for a back-to-back hvdc system," Power Delivery, IEEE Transactions on, vol. 27, no. 3, pp. 1538-1547, July 2012.

[15] Y. Ma, Z. Miao, V. R. Disfani, and L. Fan, "A one-step model predictive control for modular multilevel converters," in PES General Meeting Conference Exposition, 2014 IEEE, July 2014, pp. 1-5. 